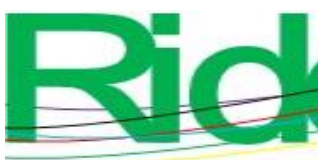

\title{
Perspectivas de la formación permanente de los docentes de educación superior
}

\section{Perspectives of the Permanent Training of Teachers in Higher Education}

\section{Perspectivas da formação permanente dos docentes da educação superior}

\author{
Marisol Esperanza Cipagauta Moyano \\ Corporación Universitaria Minuto de Dios, Colombia \\ mcipagau@uniminuto.edu.co \\ https://orcid.org/0000-0002-1378-8824
}

\section{Resumen}

La formación permanente de los docentes universitarios se ha convertido en una preocupación latente de las instituciones de educación superior. En pleno siglo XXI, en el que la incursión y constante evolución de la tecnología son innegables, resulta imperioso formular planes de acción para desarrollar las competencias docentes que demanda la era digital. En el presente artículo se abordan varios aspectos relacionados con la formación de los docentes universitarios, tal y como las características de la función mediadora, su relación con el empleo de la tecnología en el aula, las estrategias de enseñanza y de evaluación que emplean, la investigación de su práctica y su papel en la sociedad de la información y el conocimiento. El objetivo es evidenciar la importancia de la formación permanente de los docentes de educación superior, percepción que se obtiene mediante la aplicación de una encuesta a un grupo de docentes, datos recolectados a través del formato entrevista y la información recabada por medio de la revisión de la literatura existente relacionada con la temática planteada.

Palabras clave: calidad educativa, formación de docentes, función mediadora, tecnología. 


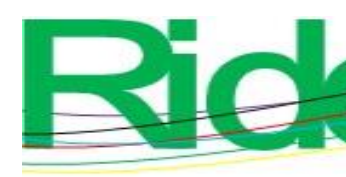

Revista Iberoamericana para la Investigación y el Desarrollo Educativo ISSN $2007-7467$

\section{Abstract}

The permanent training of university teachers has become a latent concern in higher education institutions. In the $21^{\text {st }}$ century, in which the incursion and constant evolution of technology are undeniable, it is imperative to formulate action plans to improve teaching competencies for the digital age. This article addresses several aspects related to the training of university teachers in topics like the characteristics of the mediating function, the use of technology in the classroom, teaching and evaluation strategies to be employed, research of the teaching practice, and the teachers' role in the so-called information and knowledge society. The main objective of this research is to show the importance of the permanent teachers training at higher education, perception that is gained through a survey applied to a group of teachers, collect data through a survey format and information gained through the existing literature review related to the theme raised.

Keywords: educational quality, teacher training, mediating function, technology.

\section{Resumo}

A formação permanente dos docentes universitários tem-se convertido em uma preocupação latente das instituições de educação superior. Em pleno século XXI, em que a incursão e a constante evolução da tecnologia são inegáveis, resulta imperioso formular planos de ação frente as competências do professor na era digital, justamente neste momento em que seu papel tem mudado ou reconfigurado: deixando de ser um transmissor do conhecimento para ser um mediador entre o currículo e o processo de ensino-aprendizagem. Em termos de qualidade, o desenvolvimento profissional é um dos indicadores a ser avaliado pelos organismos credenciadores e, por isso, as instituições devem contemplar toda a carreira docente dos profissionais, os quais devem incluir também a formação em outros tipos de competência, dentre elas se destaquem: trabalho em equipe, criatividade, comunicação efetiva e pensamento crítico, indo dessa maneira além das atualizações importantes na sua área do conhecimento. Em conjunto com as competências digitais, como seu uso e apropriação no desenvolvimento do ato educativo, o presente artigo aborda vários aspectos relacionados não apenas à formação dos docentes universitários - como as características da função mediadora -, mas também apresentam relação com o emprego da tecnologia na aula e as estratégias de ensino empregadas agregadas a essas facilidades; por fim, relaciona-se à 


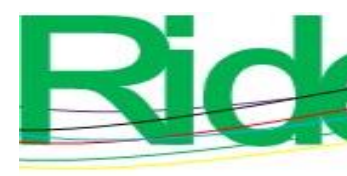

Revista Iberoamericana para la Investigación y el Desarrollo Educativo

ISSN $2007-7467$

investigação de sua prática e seu papel na sociedade da informação, dando-se a devida importância ao conhecimento digital do qual a educação não é alheia, tampouco a detentora de sua totalidade, mas intermediária na utilização daquilo que lhe for propício à transformação do saber humano. O objetivo da pesquisa é evidenciar a importância da formação continua dos docentes do ensino superior, alvo que se alcança através da realização de um inquérito a um grupo de professores, dados coletados no formato de entrevista e informações coletadas através de revisão da literatura existente relacionada à questão levantada.

Palavras-chave: qualidade educacional, treinamento de professor, função mediadora, tecnologia.

Fecha Recepción: Mayo 2020

Fecha Aceptación: Octubre 2020

\section{Introducción}

El presente artículo muestra los resultados de un trabajo investigativo sobre la importancia de la formación docente permanente en profesores que se dedican a prestar sus servicios en el nivel de educación superior, particularmente en una maestría en educación impartida en modalidad virtual. Para llevar a cabo procesos de formación docente, hay que tener en cuenta varios aspectos que apuntan al fortalecimiento de la práctica pedagógica y a la cualificación de los profesores, lo que redunda en el mejoramiento de la calidad educativa en cualquier institución. La pesquisa se realiza a partir de la literatura y estudios hechos en los últimos cinco años. También se toman en cuenta los resultados obtenidos luego de aplicar una encuesta a un grupo de docentes de una maestría en educación de una institución privada en Colombia para conocer su percepción sobre el tema de la formación docente, así como una serie de entrevistas realizadas a directivos que lideran el proceso formativo en la institución citada.

Los autores referenciados como teóricos son expertos en la materia y han dedicado su vida profesional a investigar y escribir sobre conceptos relacionados con la formación docente y las acciones que se emprenden alrededor de este proceso. Asimismo, el artículo contiene apartados específicos en los que se hace una reflexión y un análisis de las implicaciones que tiene un proceso de formación docente en las instituciones de educación superior: cómo se concibe, quiénes son sus protagonistas, quiénes son los responsables de llevarlo a cabo, de hacerle seguimiento y evaluarlo, qué beneficios representa en términos de 


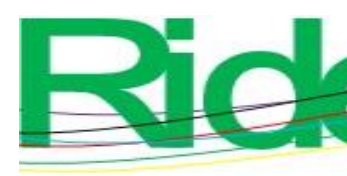

\section{Revista Iberoamericana para la Investigación y el Desarrollo Educativo ISSN $2007-7467$}

calidad para las instituciones y cómo la incursión y apropiación de la tecnología influyen en la formación de estos docentes.

Para una comprensión y análisis más amplio de la temática se revisa cómo la formación permanente de docentes es importante para una institución y su comunidad académica; la injerencia de la función mediadora del docente entre el currículo y la enseñanza; la relación entre el proceso de enseñanza y el uso y apropiación de la tecnología en el ambiente de aprendizaje; el papel de la institución y su compromiso con la formación docente; los desafíos a los que se enfrenta el docente en la propia dinámica de su ejercicio, y, por último, la evaluación de su desempeño.

El artículo finaliza con las conclusiones a las que se llega después de la correspondiente revisión de la literatura disponible y los resultados de la encuesta aplicada al grupo de profesores del programa académico mencionado y las entrevistas realizadas.

¿Por qué es importante la formación permanente de los docentes?, ¿qué papel juega la institución?, ¿cómo entender la función mediadora del docente?, ¿cuáles son esas competencias indispensables para ejercer la función docente? Para estas y otras preguntas se presenta una serie de aproximaciones desde una perspectiva del mejoramiento de la calidad en el proceso de enseñanza, aprendizaje y evaluación que desarrollan los docentes, particularmente en la educación superior.

\section{Método}

Para la búsqueda de la información y datos que soportan la investigación, se realiza una revisión de la literatura de los últimos cinco años. Se trata de un análisis de textos de libros y artículos científicos que resultaron de investigaciones sobre formación docente en el nivel universitario. A partir de esta revisión se hace una reflexión sobre los aspectos que se consideran imprescindibles a la hora de emprender procesos de formación docente universitaria y la forma en cómo las instituciones incorporan en sus planes de desarrollo este concepto.

Asimismo, se incluyen los resultados de una encuesta realizada a un grupo de profesores que ejercen su labor en una maestría en educación de una universidad privada colombiana. En dicha encuesta se indaga sobre aspectos relacionados con la formación docente, desde la propia concepción que tienen del término, la responsabilidad de la formación del docente por parte de la institución, la periodicidad de la formación y los 


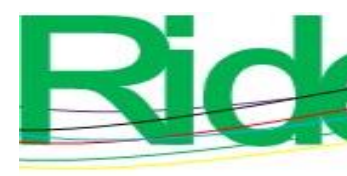

Revista Iberoamericana para la Investigación y el Desarrollo Educativo

ISSN $2007-7467$

contenidos generales que esta debe tener. También se toman en cuenta entrevistas hechas a directivos.

Los objetivos de la investigación sirvieron como base para definir las categorías conceptuales que se encuentran en el presente artículo y fueron el sustento para resignificar la importancia de la formación permanente de los docentes universitarios, que día tras día se enfrentan a nuevos retos impuestos no solo por la demanda del mercado, sino por la incursión de la tecnología en la educación y por las políticas estatales que buscan el mejoramiento de los procesos educativos en materia de formación docente. Para el caso colombiano, específicamente se hace referencia al decreto 1330 de 2019 (República de Colombia, 25 de julio de 2019).

A partir de esta metodología de tipo cualitativo, se presentan los resultados obtenidos de los instrumentos aplicados. Y a la luz de estos, se analizan las diferentes diversas categorías definidas. Finalmente, en el apartado de discusión, se hace una serie de propuestas en cuanto a estrategias de formación para docentes que laboran en educación superior.

\section{Resultados}

La encuesta hecha al grupo de docentes que prestan sus servicios en la maestría en educación en modalidad virtual, 52 en total, se hizo empleando un formulario en línea, cuyas preguntas fueron validadas previamente por expertos en educación virtual y con trayectoria en formación de docentes. Por otro lado, se entrevistó a la subdirectora general de docencia de la institución universitaria, quien respondió a una serie de cuestionamientos sobre el tema abordado. Las respuestas se incluyen a lo largo del artículo.

Frente a la pregunta de si hoy en día el docente cumple un papel de transmisor de conocimientos, un mediador del proceso de enseñanza y aprendizaje o ambos, los profesores encuestados respondieron de la siguiente manera: $85 \%$ un mediador del proceso de enseñanza y aprendizaje y $15 \%$ un transmisor de conocimientos y un mediador del proceso de enseñanza y aprendizaje. Ninguno se inclinó por la respuesta de que el docente del siglo XXI es únicamente un transmisor del conocimiento, lo que refleja el cambio de mentalidad con respecto al rol del docente en la era digital, que se considera en esencia un mediador entre el currículo y la propia enseñanza.

Bajo este panorama, entran en juego otros factores que antes no eran tenidos en cuenta porque simplemente no tenían injerencia en el quehacer docente. De acuerdo con Castillo 


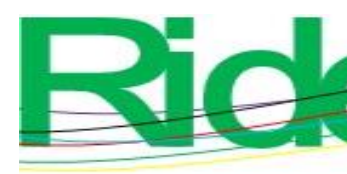

Revista Iberoamericana para la Investigación y el Desarrollo Educativo

ISSN $2007-7467$

(2017), "la calidad de una institución educativa universitaria depende fundamentalmente de la calidad humana, pedagógica y científica de sus docentes por eso ha sido una constante preocupación de las universidades la preparación y capacitación de sus profesores” (p. 67). Solo por mencionar algunos, está el hecho de que el docente debe desenvolverse hoy en día en un mundo digitalizado. En efecto, la educación no es ajena a los procesos de innovación tecnológica acaecidos en los últimos años; antes bien, se vale de la tecnología para acercar a las personas y así estas puedan estudiar incluso sin necesidad de desplazarse de un lugar a otro.

Ahora bien, frente a la discusión de si un plan de formación docente debe estar relacionado con el currículo, con los intereses de la dirección o con los intereses del docente, del total de los encuestados, $85 \%$ considera que con el currículo, $10 \%$ con los intereses del profesor y $5 \%$ con los intereses de la dirección. En este sentido, se considera que el plan debe ser coherente con la cultura organizacional de la institución y su proyección en el campo educativo.

Como se ha mencionado anteriormente, un plan de formación contempla varias acciones encaminadas a fortalecer la práctica docente y la mejora en la calidad de la educación. Al respecto, $100 \%$ de los profesores encuestados están de acuerdo con incluir temas relacionados con su disciplina y pedagogía. Esta afirmación tajante coincide con el hecho de que $85 \%$ de los encuestados considera que un plan de formación docente es útil para mantener actualizado al profesor en su saber específico y en pedagogía y $15 \%$ para cumplir con indicadores de calidad.

Al indagar sobre el concepto de formación docente, estas son algunas de las definiciones expuestas por los participantes:

- $\quad$ Es ese proceso permanente de búsqueda de conocimientos que permite fortalecer la práctica docente y responder con calidad a las demandas educativas de los nuevos sujetos. Esto reconociendo que el docente es el "eterno aprendiz" y partiendo de la base de que son las instituciones las que deben impulsar todos estos procesos formativos.

- $\quad$ Proceso permanente tanto del profesor como de la institución educativa que mantiene actualizados teórica, metodológica y pedagógicamente los procesos educativos e investigativos, lo cual redunda en la formación de sus alumnos y aporta a la institución y al país. 


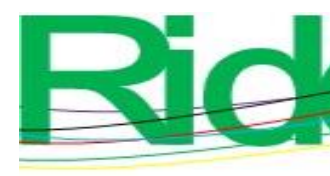

Revista Iberoamericana para la Investigación y el Desarrollo Educativo ISSN $2007-7467$

- $\quad$ Acción de cualificación personal y profesional con el objetivo de optimizar la labor docente, que a su vez redundará en la formación de mejores profesionales y programas.

- $\quad$ Proceso de continua investigación, exploración y creación guiado por un experto para pensar y desarrollar otras formas de desarrollo profesoral y profesional.

Ahora bien, teniendo en cuenta las dinámicas de la educación del siglo XXI y las perspectivas en este campo, en donde la presencia de la tecnología se incrementa a medida que pasa el tiempo, los tipos de formación se reconfiguran constantemente. Al respecto, Yadira Sánchez Velandia, subdirectora general de docencia de la Corporación Universitaria Minuto de Dios (Uniminuto), afirma: “Los profesores de educación superior deben cualificarse en aspectos como profundización disciplinar, pedagogía, didáctica y currículo, manejo de TIC y manejo de una segunda lengua" (entrevista personal, 25 de febrero de 2020). En suma, hablar de un tipo de formación tradicional es quedarse corto. En cambio, resulta interesante mencionar un tipo disruptivo, que permita hacer cosas diferentes; un tipo humanista, que dé importancia a la formación en valores; otro puede ser un tecnológico, donde haya mediación pedagógica en la apropiación de la tecnología; y por último, un tipo conservador, capaz de aceptar otras formas, pero manteniendo lo normativo y reglamentario.

El papel que representa el docente en el proceso educativo es protagónico y fundamental, constituye la base para el desarrollo del proceso educativo, por la responsabilidad que tiene de educar, no solo pensando en formar a profesionales, sino también en formar personas, lo cual quiere decir que su labor no se limita. Actualmente, el profesor se define como un mediador del proceso de enseñanza, aprendizaje y evaluación que se apropia del discurso educativo y lo materializa en la gestión del conocimiento: concede a sus alumnos la tarea de aprender y poner en práctica lo aprendido. "El rol del profesor de la sociedad del conocimiento ya no es el de uno que 'dicta su clase', sino que es un guía que va enseñando y mostrando el camino para que el alumno llegue al aprendizaje” (Castillo, 2017, p. 68).

Finalmente, en el proceso de formación docente varios son los factores a tener en cuenta. El acompañamiento que se haga antes, durante y después de la formación para evaluar las acciones correctivas y fortalecer las que se requieran es imprescindible. Imbernón (2017) menciona que "el profesorado sabe que ha de estar en constante cambio y renovación, y que la incertidumbre forma parte desde hace tiempo de su entorno profesional” (p. 22). La motivación es clave en la formación por cuanto crea la empatía necesaria para obtener buenos 


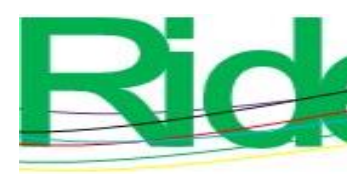

Revista Iberoamericana para la Investigación y el Desarrollo Educativo

ISSN 2007 - 7467

resultados con el estudiantado. El apoyo por parte de la institución a la formación autónoma que realice el docente, y que aporte al mejoramiento de la calidad de la educación en su contexto inmediato. Asimismo, la movilidad profesoral es un factor que incide en la formación docente, con el fin de facilitar intercambios para el desarrollo de proyectos inter y transdicisplinarios.

De acuerdo con la metodología empleada para la investigación, se identificaron como categorías de análisis las siguientes: 1) la formación específica en un campo del conocimiento; 2) el rol del profesor en el ambiente de aprendizaje; 3 ) la función mediadora de la enseñanza; 4) la apropiación de la tecnología dentro del acto educativo; 5) el compromiso de la institución; 6) los desafíos para un mundo digital, y 7) la evaluación de la docencia.

Es de suma importancia que las instituciones de educación superior contemplen siempre en sus planes de desarrollo la inversión en el desarrollo profesoral, tanto disciplinar como en otras competencias, de acuerdo con las necesidades y contextos actuales. Esto ayuda a mejorar la calidad de la enseñanza y, por consiguiente, la preparación de los futuros egresados de los programas donde los profesores imparten sus asignaturas.

Un aspecto importante a revisar es la necesidad de la formación docente de quienes se vinculan inicialmente como profesores de educación superior. Normalmente se identifican profesionales de diferentes áreas pero sin la suficiente o a veces nula preparación pedagógica para asumir la tarea de enseñar en el nivel superior. En este sentido, resulta un desafío para las instituciones de educación superior formar a estos profesionales que, si bien cuentan con las destrezas y conocimientos en su área disciplinar, requieren de una formación pedagógica sólida que los prepare para asumir el proceso de enseñanza y aprendizaje en la universidad.

La docencia entendida como vocación se reconoce en el ejercicio mismo de esta, por tanto, es pertinente indagar la motivación que lleva a una persona a convertirse en un profesional de la educación. Y en este campo se encuentran aquellos que estudiaron para ser profesores, los profesionales de distintas disciplinas que descubrieron en la docencia su sustento de trabajo y los que llegaron a ser profesores porque se dieron cuenta que tenían la pasión por enseñar y compartir con otros. En cualquiera de estos tres escenarios en los que se puede encontrar un docente, se requiere de un plan de formación permanente tanto en su disciplina como en competencias acordes con el contexto donde se desenvuelve y las condiciones necesarias para que ejerza su labor con éxito. 


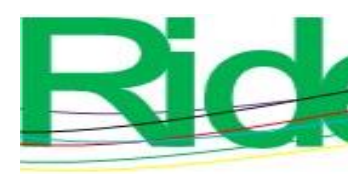

Revista Iberoamericana para la Investigación y el Desarrollo Educativo ISSN 2007 - 7467

Esta preocupación de las instituciones de educación superior ha llevado a crear programas de formación permanente, incluso centros de formación dedicados al desarrollo profesoral, que mediante acciones puntuales y temas específicos ofertan este tipo de formaciones con base en las necesidades particulares de cada universidad y contexto. Estos programas tienen como principales objetivos reflexionar sobre las prácticas en el aula, potenciar el trabajo en red y generar estrategias para adaptar los cambios en la educación superior que estén relacionados con la función docente.

\section{Discusión}

La educación del siglo XXI plantea nuevos desafíos para los docentes no solo en la adquisición de conocimientos en su área de desempeño, sino por el hecho de que su rol ha cambiado: pasó de ser el docente que tenía todo el saber y lo transmitía a ser un guía del proceso de enseñanza y aprendizaje, lo cual significa un cambio también en la forma de cómo se evalúa su oficio desde la perspectiva del alumno. "La evaluación es una oportunidad para aclarar qué es lo que se quiere del profesor y definir cuáles son las condiciones necesarias que podrían hacer posible el cumplimiento de su labor” (Rueda, 2018, p. 157).

\section{La formación permanente}

En la educación superior se encuentran profesionales de un área del conocimiento que decidieron dedicarse a la docencia; otros se han preparado para cumplir su labor como profesores, es decir, tienen de base las competencias pedagógicas necesarias para asumir este rol. Frente a este panorama, tanto para los profesionales como para los educadores se hace necesario contar con la permanente actualización de su saber, y aunque las bases teóricas no han cambiado, indistintamente del área de conocimiento, la dinámica de la educación sí, en gran medida debido a la tecnología que avanza y evoluciona cada día y permea los procesos de enseñanza, aprendizaje y evaluación. En este sentido, Vergara (2017) dice que "la formación docente tiene una finalidad innovadora. No se pretende solo poner al día al profesor en el contenido de su asignatura, sino también en el dominio de los métodos y técnicas que ha de emplear" ( p. 47). 


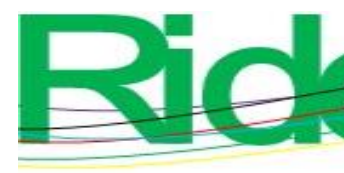

Revista Iberoamericana para la Investigación y el Desarrollo Educativo ISSN 2007 - 7467

Formar a los docentes implica, para las instituciones de educación superior, concretar planes de acción a corto, mediano y largo plazo, esto con el objetivo de estar en permanente actualización en su saber disciplinar y en las estrategias de enseñanza de acuerdo con las condiciones de los mismos ambientes de aprendizaje y el tipo de alumnos con los que se comparte. Como dice Imbernón (2017): "Todo ello ha de contribuir a un futuro donde la formación permanente del profesorado sea una verdadera herramienta de mejora, de desarrollo, de innovación y de intercambio entre el profesorado" (p. 78).

Ahora bien, independientemente de la modalidad de estudio, presencial o a distancia, la formación permanente de los docentes ha de ser una constante porque se requiere siempre mejorar la calidad de la enseñanza, y esto solo se logra si se hace un trabajo conjunto entre los involucrados y responsables de los procesos educativos, por tanto, es necesaria la inversión. Al respecto, Santos (2017) sostiene lo siguiente:

La mejora de la calidad de la enseñanza y de la profesionalización de los docentes, exige unas condiciones organizativas que hagan viable al menos y si es posible fácil la transformación y la mejora de la racionalidad y de la justicia de la práctica educativa (p. 51).

Si bien hoy en día se encuentran formaciones online, muchas de ellas sin costo alguno, con las que los docentes se pueden actualizar tanto en su saber específico como en temas relacionados con la enseñanza, siempre será necesario acudir a otras posibilidades de formación presencial, con expertos temáticos que preparen a los profesores para enfrentar el mundo complejo y cambiante de la educación superior.

La formación no para, avanza al ritmo de la evolución de la educación y las tendencias con el propósito de estar a la vanguardia, de ofrecer un servicio de talla mundial a personas que cada vez más se integran y conectan haciendo uso de la tecnología y sus ventajas.

\section{La función mediadora}

La figura del docente siempre se había visto como el transmisor del conocimiento; con la llegada del Internet, esto cambió y asumió un papel de mediador de este. El hecho de encontrar información por todas partes y a un clic: las posibilidades son infinitas; pero no todas las fuentes son confiables. Es en este punto donde el docente entra a escena: al mediar ayuda a seleccionar y filtrar la información útil. De acuerdo con Castilla (2018), "el rol del profesor de la sociedad del conocimiento ya no es el de uno que dicta su clase, sino que es 


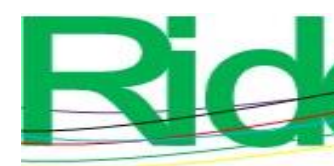

Revista lberoamericana para la Investigación y el Desarrollo Educativo

ISSN 2007 - 7467

una guía que va enseñando y mostrando el camino para que los alumnos lleguen al aprendizaje" (p. 68).

Por eso la figura del docente es importante en la educación, incluso si se acude al autoaprendizaje, pues detrás de los diseños curriculares ha estado una persona con la suficiente preparación y competencias en el desarrollo e implementación de cualquier curso, así las prestigiosas universidades ponen a disposición formaciones en línea para quienes desean conocer sobre una temática o quieran actualizar sus conocimientos.

Al hacer referencia a una función mediadora del docente, esta incluye una serie de características que la identifican como tal, lo que lleva al profesor a convertirse en arquitecto, constructor, puente, motor, transformador, adaptador, amplificador, entrenador, filósofo, terapeuta, guía, gestor, lanzador, brújula, chef, médico, samaritano, director y actor, como lo afirma Tébar (2017, p. 7).

Esta función mediadora del proceso de enseñanza y aprendizaje requiere de la permanente formación de los docentes, de manera especial en competencias digitales, que lo preparen para hacer búsquedas efectivas y confiables en la Red, el manejo de herramientas tecnológicas y la conformación de redes y alianzas como comunidades de práctica o de aprendizaje que facilitan la gestión del conocimiento y de la información. "Los propios docentes constituyen uno de los factores más importantes que influyen en la cuestión de cómo enseñar. Dirigir el aprendizaje de los estudiantes en cualquier nivel es una empresa personal” (Eggen y Kauchak, 2015 p. 33).

El profesor, en su función mediadora, aporta con su saber y su experiencia al desarrollo del proceso de enseñanza, aprendizaje y evaluación, y va más allá de una simple transmisión de conocimientos, como tradicionalmente lo era; es partícipe activo junto con sus alumnos en todo lo que implica el acto educativo.

\section{La enseñanza y la tecnología}

Con la aparición de la Internet el mundo cambió, y la educación también comenzó a hacerlo paulatinamente, incorporando la tecnología al proceso de enseñanza y aprendizaje. En esa línea, los planes de formación de los docentes en las distintas instituciones de educación superior han incluido programas de competencias digitales. Y es que es necesario que las instituciones lo hagan, pues cada vez más se impone la cultura digital. 


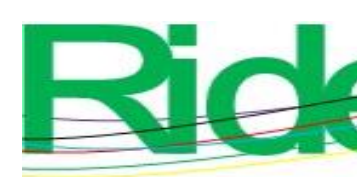

Revista Iberoamericana para la Investigación y el Desarrollo Educativo ISSN 2007 - 7467

La tecnología está dando lugar a grandes cambios en la economía, en la forma en que nos comunicamos y nos relacionamos unos con otros, y cada vez más en la forma en que aprendemos. Sin embargo, nuestras instituciones educativas fueron creadas en gran medida para otra época, en torno a una actividad industrial en lugar de una era digital (Bates, 2017, p. 21).

Son varios los ejemplos de universidades que ofertan programas en línea, cursos virtuales y todo un abanico de posibilidades que solo requieren contar con conexión a internet y del aprendizaje autónomo del participante. Pero detrás de todo este trabajo están diseñadores instruccionales, pedagogos, comunicadores, diseñadores gráficos, programadores web, correctores de estilo, todo un equipo interdisciplinario que desarrolla los contenidos y los pone a disposición de los interesados.

La relación entre enseñanza y tecnología demanda de los protagonistas del acto educativo un cambio de mentalidad. Los docentes deben tener en cuenta que ya no son los únicos que poseen la información, sino que esta se encuentra por todos partes, a un simple clic, por eso ese papel de transmisor del conocimiento se revalúa; ahora, como hemos indicado, es un mediador del aprendizaje, un guía, un acompañante, o si se le quiere llamar, un mentor que está al lado del alumno y que aprende con él. Al respecto, Yadira Sánchez Velandia, subdirectora general de docencia de la Uniminuto sostiene:

La tecnología es la herramienta que puede facilitar los procesos de aprendizaje, y en la medida que se apropie por parte de los estudiantes y los profesores se podrán generar procesos de mediación de mayor impacto. La metodología virtual exige un dominio de las tecnologías, pues es a través de estas que se puede promover el aprendizaje autónomo y el acceso a la ciencia y la innovación (entrevista personal, 25 de febrero de 2020).

Del otro lado del escritorio se encuentra el alumno hiperconectado que se desenvuelve a diario en un mundo digital, por lo que su proceso educativo ha de estar alineado con la tecnología. Es común el ejemplo del uso del celular en una clase: algunos docentes lo ven como un enemigo, otros lo ven como un aliado del proceso de enseñanza-aprendizaje. Se trata, pues, de usar y apropiar la tecnología para estar cerca de los alumnos y hacerlos copartícipes de su propio aprendizaje. "La tecnología podrá sustituir texto y cambiar formas de aprender, pero difícilmente sustituirá totalmente al profesorado, ayudando a aprender a separar el grano de la paja" (Imbernón, 2017, p. 44). 


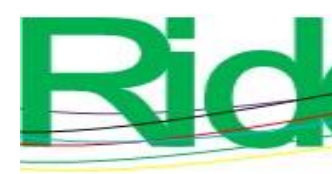

Revista Iberoamericana para la Investigación y el Desarrollo Educativo

ISSN 2007 - 7467

Un aspecto también a tener en cuenta es el papel del docente como innovador pedagógico; requiere estar en formación continua sobre tendencias educativas, metodología de enseñanza, estrategias de evaluación, gestión de la investigación y aspectos relacionados con la internacionalización del currículo que le permitan compartir con otros su saber y enriquecerse a través del trabajo colaborativo.

\section{La institución}

Y ¿cuál es la responsabilidad de la institución en la formación docente? Por lo general, la institución contempla en sus planes de desarrollo temas relacionados con la formación de sus profesores. Y es que debe hacerlo, pues necesita garantizar y mantener la calidad de la enseñanza que se imparte dentro de sus aulas, ya sean materiales o virtuales, y esto solo se logra si se invierte en su capital humano; la institución que no lo hace corre el riesgo de quedar rezagada frente a las demás. Invertir en infraestructura física y en tecnología sin hacerlo en la formación de los docentes no sirve de nada, pues se necesita del talento de estos para operar y hacer uso apropiado de una y otra. "La institución deberá desarrollar políticas y mecanismos para atraer, desarrollar y retener el talento humano acorde con su misión" (República de Colombia, 25 de julio de 2019).

En este orden de ideas, el mismo decreto dice:

La institución deberá establecer en el programa las estrategias para la formación en investigación creación que le permitan a profesores y estudiantes estar en contacto con los desarrollos disciplinarios e interdisciplinarios, la creación artística, los avances tecnológicos y el campo disciplinar más actualizado, de tal forma que se desarrolle el pensamiento crítico y/o creativo (República de Colombia, 25 de julio de 2019).

Desde la perspectiva normativa se tendría a la institución como la única responsable del tema de formación de los docentes; pero existe también la inquietud por parte de estos, y es que lo asuman como reto personal. Esto se evidencia en una encuesta aplicada a una muestra de 24 docentes de una maestría en modalidad virtual de una institución privada en Colombia, que forma parte de la presente investigación: $90 \%$ considera que la responsabilidad de la formación docente es un tema compartido entre la institución y el profesor, y el resto, $10 \%$, que le corresponde exclusivamente al profesor. Como se puede apreciar, ninguno consideró que sea solo responsabilidad de la institución. Estos datos 


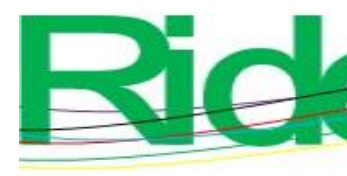

\section{Revista Iberoamericana para la Investigación y el Desarrollo Educativo ISSN 2007 - 7467}

demuestran el trabajo colaborativo que hay entre institución y profesor, dos actores que consideran importante hacerlo así.

La iniciativa de la formación puede partir de los beneficiarios, es decir, de los propios docentes, o bien desde la dirección. La clave está en asegurar la calidad y pertinencia de estos procesos, todo lo cual redunde en beneficios para el proceso de enseñanza, aprendizaje y evaluación de los alumnos. Por tanto, las competencias que el docente desarrolle o fortalezca han de estar relacionadas con estos tres componentes. "La disposición del profesor para aprender constituye un principio sin el cual cualquier intento por ofrecer formación a los docentes sería en vano, es indispensable que este interés exista” (Cipagauta, 2017, p. 50).

Con las particularidades de cada institución, se requiere de una formación constante de los docentes, pues son estos, en última instancia, quienes ponen en práctica las acciones definidas en los ambientes de aprendizaje y establecen la relación con los alumnos, los primeros y directos beneficiarios. Al respecto, Parra (2014) afirma que "fortalecer el desempeño profesional del docente de aula en su rol de mediador en los procesos de enseñanza y aprendizaje es de gran importancia porque le permite facilitar y promover el potencial del estudiante" (p. 178). En sintonía con lo anterior, en el caso de la Uniminuto, desde la Subdirección General de Docencia se orientan las políticas y lineamientos para los profesores; en específico, promueve el desarrollo profesoral como una estrategia de acompañamiento al profesor durante su trayectoria en la institución para garantizar su desarrollo integral, esto implica su dimensión personal, profesional y pedagógica. Finalmente, en materia de formación, el compromiso resulta compartido.

Se necesita de la participación activa de todos los actores del proceso de enseñanza-aprendizaje, donde cada quien asuma su rol y lo interprete de la mejor manera: que los directores con una gran identidad por su escuela, así como un liderazgo efectivo, dirijan a sus cuerpos docentes para formar una plantilla que trabaje colaborativamente para conseguir el mismo objetivo, para ello se debe reconocer que existen problemas dentro de la institución y después elaborar un plan estratégico para contrarrestar estas problemáticas, involucrando a los alumnos, padres de familia, comunidad en general, y gobierno de los diferentes niveles (Martínez, Guevara y Valles, 2016 p. 132). 


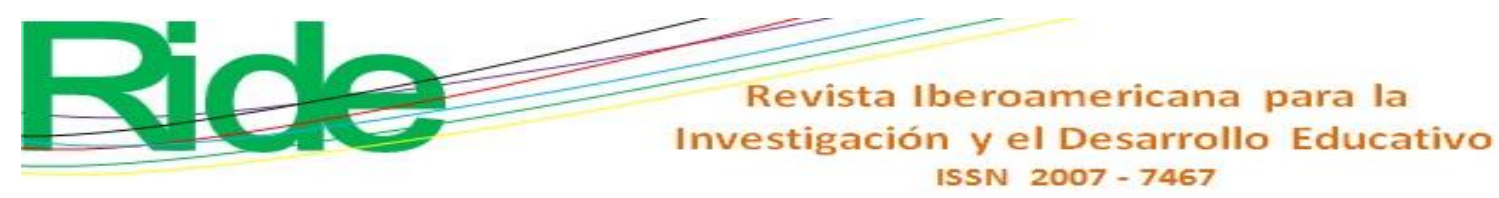

\section{Los desafíos para el docente}

La formación docente representa tanto para la institución como para el mismo profesor un reto permanente por la misma dinámica que impone la academia. Para el docente específicamente, el estar actualizado tanto en su disciplina como en estrategias pedagógicas y técnicas didácticas, así como en el uso y apropiación de la tecnología, representan los mayores desafíos. Como afirman Glogger, Herppich y Seidel (2018): "Hoy enfrentamos la necesidad de vincular el conocimiento profesional con las acciones de los maestros en el proceso de enseñanza" (p. 176).

Un primer paso para el docente es tener conciencia de que debe estar a la vanguardia de la tendencias educativas, así como reconocer que está sirviendo de mediador entre la enseñanza y el aprendizaje de personas que luego saldrán a enfrentarse al complejo mundo laboral, cada vez más global, sin fronteras, digital e hiperconectado. El docente ha de ser de ese acompañante que ayuda a que la información se procese de forma adecuada, de tal manera que facilite la gestión del conocimiento en las diferentes áreas disciplinares.

Se necesitan métodos de enseñanza que ayuden a desarrollar y transferir las destrezas específicas tanto a los efectos del desarrollo como de la diseminación del conocimiento, y al mismo tiempo que preparen profesionales para trabajar en una sociedad basada en el conocimiento (Bates, 2017, p. 20).

Otro de los factores clave del papel del docente en el proceso de enseñanzaaprendizaje tiene que ver con la investigación de su propia práctica dentro del aula. Debe evaluar su quehacer pedagógico y a partir de los resultados diseñar mejoras en pro de la calidad de su propio desempeño, lo que redunda en el fortalecimiento de su labor. "La calidad de una institución educativa universitaria depende fundamentalmente de la calidad humana, pedagógica y científica de sus docentes, por eso ha sido una constante preocupación de las universidades la preparación y capacitación de sus profesores" (Castillo, 2017, p. 67).

Así, el docente no solo debe complir con acompañar al estudiante, sino que tiene un compromiso con la evaluación de su proceso de aprendizaje: obtener una retroalimentación de su trabajo que subraye fortalezas, debilidades y áreas de mejora, no simplemente asignar una calificación. En este aspecto, el papel del docente se complementa con el proceso evaluativo que realiza. "El último proceso clave y 'fundamental' de la enseñanza y el 


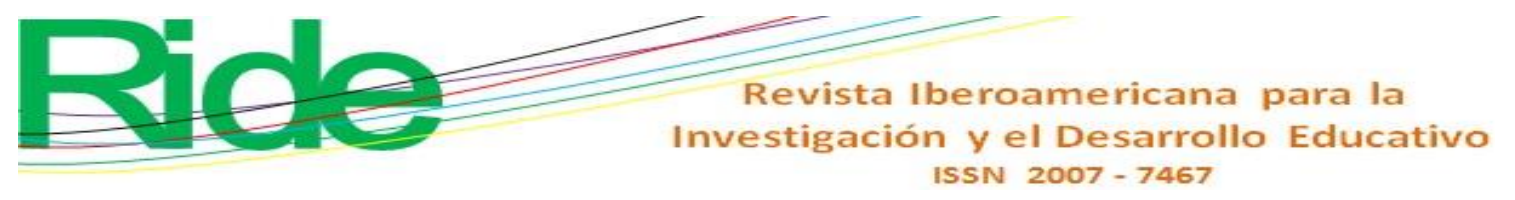

aprendizaje es la evaluación y la innovación: la evaluación de lo que se ha hecho, y luego la búsqueda de formas para mejorarla" (Bates, 2017, p. 425).

El profesor, al ser un agente activo, participativo e innovador, partiendo del hecho de que cumple con estas características en el ejercicio de su función docente, evidencia estas cualidades precisamente en el proceso de enseñanza, aprendizaje y evaluación que desarrolla con sus alumnos. "Los docentes efectivos son aquellos que logran promover el aprendizaje entre sus estudiantes" (Elacqua, Hincapié, Vegas y Alfonso, 2018, p. 6) En este sentido, incorporar nuevas técnicas didácticas, ser disruptivo, creativo y codiseñador de los contenidos promueve el desarrollo de prácticas pedagógicas que generan impacto en el aprendizaje, que se traduce en la generación y gestión de nuevo conocimiento.

En pleno siglo XXI, donde el rol del docente se reconfigura y se convierte en un mediador del aprendizaje, otras son las competencias que se deben fortalecer en su quehacer, porque la dinámica de la educación es otra, el acceso a la tecnología, su uso y apropiación demandan un docente con habilidades que respondan a estos nuevos desafíos, pero también un docente con habilidades sociales fortalecidas, pues son igualmente otras las relaciones que se establecen en el acto educativo. "Reperfilar el papel de los docentes bajo una relación diferente con el conocimiento. Una relación distinta a la que tradicionalmente habían tenido (y bajo la cual muchos fuimos formados)" (Cobo, 2017, p. 19).

\section{La evaluación de la docencia}

La evaluación docente en las instituciones de educación superior forma parte de los procesos inherentes a la actividad académica y a todos aquellos de calidad que se implementan. "Es decir, la evaluación del profesorado es útil para proporcionar información que favorezca el mejoramiento profesional del docente y la toma de decisiones a nivel administrativo" (Márquez y Madueño, 2016, p. 58).

Si bien las instituciones incluyen en estos procesos la autoevaluación del desempeño del docente y la coevaluación, la realizada por los alumnos es fundamental por cuanto son ellos los directos beneficiarios de la labor que sus docentes realizan. "La evaluación docente se caracteriza por valorar en algunos casos desde la satisfacción de los estudiantes, el desempeño de los profesores, sus habilidades, el uso de recursos psicopedagógicos, estrategias de aprendizaje e investigación, hasta su apego a políticas institucionales" (Flores, Gatica, Sánchez y Martínez, 2017, p. 97). 


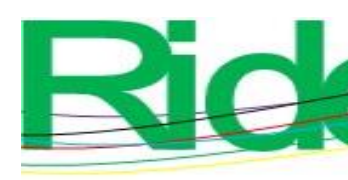

Revista Iberoamericana para la Investigación y el Desarrollo Educativo ISSN 2007 - 7467

De acuerdo con estos autores, la evaluación docente integra varios aspectos a tener en cuenta. El desempeño de este puede ser visto desde diversos ángulos y según donde se mire la percepción cambia. Así, si solo se tiene en cuenta la opinión del estudiantado, “el profesorado lo equipara a una espada de Damocles que cualquier estudiante puede calificarlo mal, sin una justificación de peso” (Arbesú y García, 2017, p. 172).

Visto de esta manera, se pierde el sentido de una evaluación transparente para el docente, por tanto, un primer reto para la institución es diseñar estrategias que permitan una evaluación justa, en donde no haya cabida a subjetividades al momento de aplicar instrumentos que permitan recoger la percepción del alumnado en cuanto al desempeño de sus docentes, pero también velar por la formación de estos para que cumplan a cabalidad su rol en el proceso de enseñanza y aprendizaje. "En suma, se debe evaluar la docencia asumiendo su complejidad, grado de idoneidad y por la pertinencia de las acciones realizadas" (Guzmán, 2016, p. 294).

No se trata de tener docentes que solo sepan de su área de conocimiento, sino de que, a través de la formación continua, sean capaces de enfrentar los desafíos de una educación dinámica y cambiante en cuanto a metodologías de enseñanza, formas de evaluar y trato con sus alumnos, incluso hacerlos partícipes del diseño mismo de los instrumentos. Así lo dicen Arbesú y García (2017): "Los expertos y directivos coinciden en que los profesores debieran ser considerados en el diseño e implementación de los procesos de evaluación docente” (p. 173). Una vez esto ocurra, las instituciones contarían con una evaluación más democrática y participativa que pueda incluir aspectos no tenidos en cuenta hasta ahora de acuerdo con la forma tradicional de hacer el proceso. "La evaluación de la actividad docente, al igual que la realizada a las instituciones, programas o procesos, no está exenta de llevar a cabo su propio proceso de valoración y permanente perfeccionamiento de la labor docente en el contexto universitario" (Ramos, Ruiz, Pulido y Marín, 2019, p. 28).

Así las cosas, la evaluación docente constituye un tema de prioridad en las agendas de las instituciones de educación superior; darle el lugar que le corresponde y hacer partícipes activos a todos los involucrados es una tarea prioritaria si se tiene en cuenta que la calidad con que este proceso se desarrolle redunda en beneficios para toda la comunidad educativa. "La calidad de la educación superior depende, entre otros factores, de la calidad de la enseñanza y, esta, a su vez, se ve fuertemente influida por la calidad del docente" (Moreno, 2018, p. 88). 


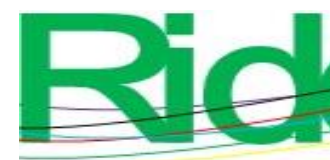

\section{Revista Iberoamericana para la Investigación y el Desarrollo Educativo ISSN 2007 - 7467}

En la tabla 1 se resumen las perspectivas propuestas para la formación permanente de los profesores que prestan sus servicios en educación superior a partir de las fuentes consultadas y analizadas.

Tabla 1. Perspectivas en la formación de un docente de educación superior

\begin{tabular}{|c|c|}
\hline Categoría & Descripción \\
\hline Formación permanente & $\begin{array}{l}\text { Los docentes de educación superior } \\
\text { requieren estar actualizados en su saber } \\
\text { disciplinar y en otras competencias para } \\
\text { afrontar las dinámicas educativas. }\end{array}$ \\
\hline Función mediadora & $\begin{array}{l}\text { Ser mediador del proceso de enseñanza } \\
\text { aprendizaje significa asumir un rol activo } \\
\text { para generar aprendizajes significativos en } \\
\text { los alumnos. }\end{array}$ \\
\hline Tecnología & $\begin{array}{l}\text { Usar y apropiar la tecnología como aliada } \\
\text { en el propio acto educativo brinda otras } \\
\text { posibilidades de avanzar y evolucionar } \\
\text { dando paso a una auténtica gestión del } \\
\text { conocimiento. }\end{array}$ \\
\hline Papel de la instituciones & $\begin{array}{l}\text { Los programas de formación docente } \\
\text { requieren del apoyo de las instituciones y } \\
\text { de un seguimiento continuo por parte de } \\
\text { estas. }\end{array}$ \\
\hline Retos de la docencia & $\begin{array}{l}\text { La dinámica de la academia invita cada día } \\
\text { a enfrentar nuevos desafíos en una era } \\
\text { como la digital, y todo lo que ella implica } \\
\text { en formación docente. }\end{array}$ \\
\hline Evaluación & $\begin{array}{l}\text { La evaluación permite crear espacios para } \\
\text { la reflexión y el mejoramiento continuo de } \\
\text { cualquier proceso. }\end{array}$ \\
\hline
\end{tabular}

Fuente: Elaboración propia 

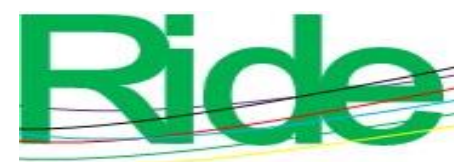

Revista Iberoamericana para la Investigación y el Desarrollo Educativo

ISSN $2007-7467$

\section{Conclusiones}

La formación docente ha de ser una constante en las instituciones de educación superior que tengan como propósito el mejoramiento de la calidad del proceso de enseñanza, aprendizaje y evaluación: los profesores han de estar actualizados tanto en su saber específico como en temas de pedagogía. En este sentido, la formación se define como un trabajo de interés mutuo en el que la institución junto con los profesores definen la ruta a seguir, dependiendo de las necesidades y del contexto educativo. Esto es evidente en la percepción que sobre temas de formación tienen los docentes encuestados.

La investigación realizada demuestra que elaborar un plan de formación docente en donde se incluyan programas de cualificación, como un proceso permanente que se relacione con el currículo, la misión y visión de la institución, promueve acciones que corresponden realmente a la proyección que la institución tenga en el presente y futuro.

Uno de los temas fundamentales para incluir actividades vinculadas con la formación docente es, sin lugar a dudas, la tecnología, pues llegó para quedarse y su evolución constante no es ajena a los procesos educativos, por tanto, el docente del siglo XXI es el docente que se mueve en lo digital, que se apropia e incorpora la tecnología al proceso de enseñanza, que, finalmente, la hace su aliada y la aprovecha en beneficio de la gestión del conocimiento y el aprendizaje significativo de sus alumnos. 


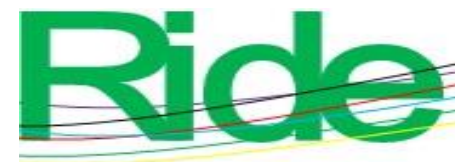

Revista Iberoamericana para la Investigación y el Desarrollo Educativo ISSN 2007 - 7467

\section{Referencias}

Arbesú, M. y García, J. (2017). La evaluación docente en jaque, la visión de los funcionarios universitarios. Revista Iberoamericana de Evaluación Educativa, 10(2), 171-185. Recuperado de https://dialnet.unirioja.es/servlet/articulo?codigo=6205629.

Bates, A. (2017). La enseñanza en la era digital. Una guía para la enseñanza y el aprendizaje. Canadá: Asociación de Investigación Contact North. Recuperado de http://www.tonybates.ca/2017/02/04/a-spanish-version-of-teaching-in-a-digital-ageis-now-available/.

Castilla, H. (2018). Más allá de los conocimientos. El arte de ser profesor mediador en el proceso de aprendizaje. Bogotá, Colombia: Uniminuto.

Castillo, V. (2017). La formación pedagógica del docente universitario. Palermo Business Review, (16), 65-73. Recuperado de https://search-proquestcom.ezproxy.uniminuto.edu/docview/2080197389?accountid=48797.

Cipagauta, M. (2017). Evaluar la docencia para mejorar la enseñanza. Bogotá, Colombia: Uniminuto.

Cobo, C. (2017). La innovación pendiente. Reflexiones (y provocaciones) sobre educación, tecnología y conocimiento. Montevideo, Uruguay: Debate.

Eggen, P. y Kauchak, D. (2015). Estrategias docentes. Enseñanza de contenidos curriculares y desarrollo de habilidades del pensamiento. Ciudad de México, México: Fondo de Cultura Económica.

Elacqua, G., Hincapié, D., Vegas E. y Alfonso, M. (2018). Profesión: profesor en América Latina. ¿Por qué se perdió el prestigio docente y cómo recuperarlo? Nueva York, Estados Unidos: BID.

Flores, F., Gatica, F., Sánchez, M, y Martínez, A. (2017). Evolución de la evaluación del desempeño docente en la Facultad de Medicina; evidencia de validez y confiabilidad. Revista Investigación en Educación Médica, 6(22), 96-103. Recuperado de https://dx.doi.org/10.1016/j.riem.2016.06.004.

Glogger, I., Herppich, S. and Sidel, T. (2018). Linking teachers' professional knowledge and teachers' actions: Judgment processes, judgments and training. Teaching and Teacher Education Review, 76, 176-180. Retrieved from https://doi.org/10.1016/j.tate.2018.08.005. 


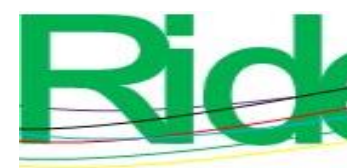

Revista Iberoamericana para la Investigación y el Desarrollo Educativo

ISSN 2007 - 7467

Guzmán, J. (2016). ¿Qué y cómo evaluar el desempeño docente? Una propuesta basada en los factores que favorecen el aprendizaje. Propósitos y Representaciones, 4(2). Recuperado de http://dx.doi.org/10.20511/pyr2016.v4n2.124.

Imbernón, F. (2017). Ser docente en una sociedad compleja. La difícil tarea de enseñar. Barcelona, España: Graó.

Márquez, L. y Madueño, M. (2016). Propiedades psicométricas de un instrumento para apoyar el proceso de evaluación del docente universitario. Revista Electrónica de Investigación Educativa, 18(2). Recuperado de http://www.scielo.org.mx/scielo.php?script=sci_arttext\&pid=S1607$40412016000200004 \& \operatorname{lng}=$ es\&tlng=es.

Martínez, G., Guevara, A. y Valles, M. (2016). El desempeño docente y la calidad educativa. Revista Ra Ximhai, 12(6), 123-134. Recuperado de https://www.redalyc.org/articulo.oa?id=46148194007.

Moreno, T. (2018). La evaluación docente en la universidad: Visiones de los alumnos. Revista Iberoamericana sobre Calidad, Eficacia y Cambio en Educación, 16(3). Recuperado de https://revistas.uam.es/reice/article/view/9715.

Parra, K. (2014). El docente y el uso de la mediación en los procesos de enseñanza y aprendizaje. Revista de Investigación, 38(83), 155-180. Recuperado de https://www.redalyc.org/articulo.oa?id=376140398009.

Ramos, A., Ruiz, L., Pulido, E., Marín, M., y Redondo, P. (noviembre, 2019). Estrés ocupacional y evaluación de desempeño en docentes universitarios del departamento del Cesar, Colombia. Revista Encuentros. doi:http://dx.doi.org.ezproxy.uniminuto.edu/10.15665/encuent.v17i01.1595

República de Colombia. (25 de julio de 2019). Decreto 1330 de 2019. "Por el cual se sustituye el Capítulo 2 y se suprime el Capítulo 7 del Título 3 de la Parte 5 del Libro 2 del Decreto 1075 de 2015 -Único Reglamentario del Sector Educación”. Colombia: Ministerio de Educación Nacional. Recuperado de https://www.mineducacion.gov.co/1759/articles-387348_archivo_pdf.pdf.

Rueda, M. (2018). Los retos de la evaluación docente en la universidad. Publicaciones. Facultad de Educación y Humanidades del Campus de Melilla, 48(1), 143-159. Recuperado de https://revistaseug.ugr.es/index.php/publicaciones/article/viewFile/7334/6419. 


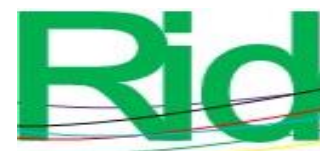

Revista Iberoamericana para la

Santos, M. (2017). La gallina no es un águila defectuosa. Bogotá: UNIMINUTO.

Tébar, L. (2017). La función mediadora de la educación. Foro Educacional, (28), 79-98.

Recuperado

de

http://ediciones.ucsh.cl/ojs/index.php/ForoEducacional/article/view/790/736.

Vergara, M. (2017). La práctica cotidiana de los profesores. Un estudio desde el proceso de formación en el posgrado. Guadalajara, México: Universidad de Guadalajara. 